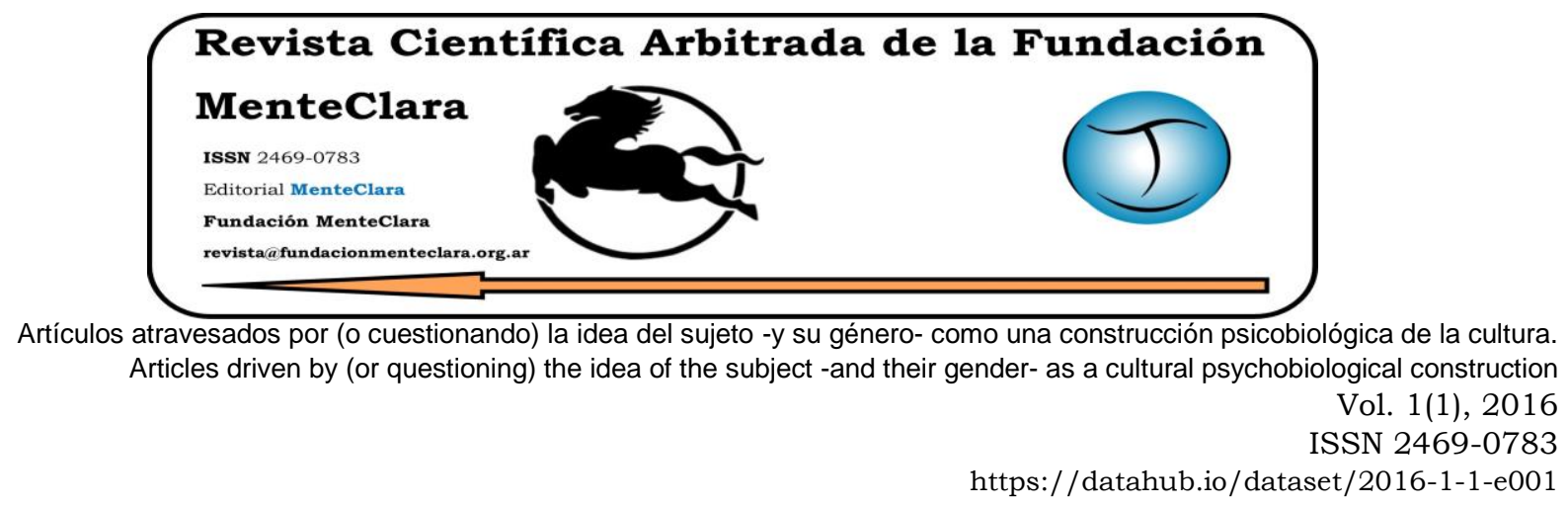

\title{
ANTONIO DE MONTSERRAT - LA RUTA DE LA SEDA Y LOS CAMINOS SECRETOS DEL TANTRA
}

\author{
ANTONIO DE MONTSERRAT - THE SILK ROAD AND THE SECRET PATHS OF \\ TANTRA
}

Oscar R. Gómez oscar@tantra.org.es

Escuela Argentina de Tantra

Cómo citar este artículo / Citation: Gómez, O. (2016). "Antonio de Montserrat La Ruta de la Seda y los caminos secretos del Tantra". Revista Científica Arbitrada de la Fundación MenteClara, Vol 1 (1), 5-20. DOI: 10.32351/rca.v1.1.8

Copyright: (C) 2016 RCAFMC. Este artículo de acceso abierto es distribuido bajo los términos de la licencia Creative Commons Attribution-Non Commercial (by-cn) Spain 3.0. Recibido: 01/6/2015. Aceptado: 01/01/2016 Publicación online: $20 / 01 / 2016$

\section{Resumen}

En este artículo se presenta la biografia de Antonio de Monserrat con el objeto de insertar en el pensamiento crítico budista a quien se considera el primer occidental iniciado en la filosofia tántrica e impulsor de ésta en Occidente a través de la Compañía de Jesús. Para ello, primero se hace un recorrido histórico que pone en foco cómo el budismo es desplazado de la India y se refugia entre las poblaciones de Asia central como la etnia Uigur en la actual Turquestán, cómo es adoptado por los emperadores chinos y se expande a lo largo de toda la Ruta de la Seda. La combinación del budismo indio con influencias occidentales (grecobudismo) dio origen a diversas escuelas budistas en Asia Central y en China. Luego se caracteriza en forma sintética la versión esotérica que adquiere el budismo (el tantra) y que se consolida en el siglo VIII en el Tíbet como budismo vajrayana (tántrico).

Ésta es la forma de budismo que toman los gobernantes, que promueve la igualdad completa de personas y género, la idea del sujeto como una construcción de la cultura y la noción de deidades metafóricas -útiles para modelar el carácter de las personas pero de absoluta inexistencia- además del postulado budista de verdad relativa. Esta visión no teísta —o transteísta, como Gómez la prefiere llamar- se reflejaba en la total tolerancia religiosa del imperio Chino, Uigur y Mongol, que garantizaba la seguridad y el libre intercambio por la Ruta de la Seda. Es esta visión de sujetos no divididos en castas ni diferenciados por sangre lo que maravilla a de Montserrat al decir que los tibetanos "no tienen reyes entre sî" e inflama la avidez de quienes viajaron especialmente (a partir de los escritos de éste) a iniciarse en el budismo tántrico tibetano como los jesuitas Antonio de Andrade y Juan de Brito. El tercer 
apartado se dedica de lleno a la biografia de Antonio de Monserrat y a precisar su contacto con el tantra.

\begin{abstract}
This article presents Antonio de Montserrat's biography to insert him in Buddhist critical thinking as whom is considered the first Westerner initiated into tantric philosophy and who became a driver thereof in the West through the Society of Jesus. To do so, a historical review is first presented to focus on the way Buddhism was removed from India and found refuge among the peoples of Central Asia such as the Uyghurs in present-day Turkistan, how it was then adopted by Chinese emperors and spread throughout the Silk Road. The combination of Indian Buddhism and Western influences (Greco-Buddhism) gave rise to several Buddhist schools in Central Asia and China. Then, the esoteric form Buddhism took (tantra) is briefly described, which was consolidated as Vajrayana (tantric) Buddhism in Tibet in the eighth century.

That is the Buddhist form rulers have adopted, which promotes full social and gender equality, the idea of the subject as a cultural construction and the notion of metaphorical deities - useful to model people's character but completely nonexistent - in addition to the Buddhist principle of relative truth (not absolute). This non theistic view —or transtheistic, as Gómez would rather call, was projected in the absolute religious tolerance within the Chinese, Uyghur, and Mongolian empires, which ensured safety and free exchange on the Silk Route. Such standpoint of people not divided into castes or differentiated by reason of bloodline is what amazes de Montserrat when saying Tibetans "have no kings among them" and what encourages those who made a journey (based on de Montserrat's writings) especially to receive initiation into Tibetan Tantric Buddhism such as Jesuits Antonio de Andrade and John de Brito. Finally, the article jumps in Antonio de Montserrat's biography and it shows its connection with tantrism.
\end{abstract}

\title{
Palabras Claves/ Keywords
}

Tantra; Tantrismo; Budismo; Sociologia; Antropología; Religión; Tantrism; Buddhism; Sociology; Anthropology; Religion; Tantric Studies 


\section{El Budismo en Asia central ${ }^{1}$}

Muchas personas están convencidas de que la luz viene de Oriente, casi la sociedad occidental así lo cree; y en esa creencia, Oriente parecería ser la India al punto que a partir de los movimientos New Age se introdujeron de ahí un sinnúmero de técnicas para alcanzar una supuesta iluminación que consiste en sumergir la consciencia en planos oníricos (Urban, 1999).

Es decir, ver la "realidad" que el sistema hindú utilizó para someter a sus habitantes a la más impiadosa esclavitud y servilismo. Una especial clase de "opio" ideológico que posibilita que los parias sonrian en la indignidad a la que están sometidos y la acepten como consecuencia de sus vidas pasadas, karma.

Sin embargo, ciertamente la luz llega de Oriente. No solo porque desde ese punto emerge el sol sino también porque la luz intelectual ingresó a través de la Ruta de la Seda.

Esta ruta es el resultado de la curiosidad del emperador chino Wu, de la dinastía Han y que gobernó de 141 a 87 a. C., por los pueblos civilizados que estaban más allá de las tribus bárbaras en Occidente (Bellec, 2003).

Esta dinastia, que hablaba lenguas sino-tibetanas, se extendió hasta las tribus nómades occidentales y de ahí a las llanuras húngaras y hasta los montes Cárpatos.

El emperador Wu envió en el año 138 a. C. una embajada con el objetivo de conseguir una alianza militar con poblaciones indoeuropeas (entre ellos la etnia Uigur) para frenar el poder y agresividad de los xiongnu - tribus nómadas asentadas en la actual Mongolia- que amenazaban constantemente la seguridad de China.

Esta embajada, que no logró ninguna alianza militar, informó de la existencia de muchos reinos. Algunos de estos reinos eran verdaderas potencias militares como Persia, Caldea y el Imperio Romano con las que la embajada sí se relacionó y se produjo un intercambio de bienes y conocimientos.

\footnotetext{
${ }^{1}$ En este trabajo se ha evitado el uso de un sistema fonético para los términos de origen sánscrito, mongol o tibetano ya que considero que la pronunciación de las palabras en sí mismas carecen de sentido si el sujeto hablante desconoce su significación. Ese saber puede ser de utilidad para los estudiosos históricos o eruditos pero esta biografia está destinada exclusivamente para reivindicar al que considero el primer occidental iniciado en la filosofia tántrica y el impulsor de ésta en Occidente.
} 
Es así cómo esta misión comenzó a trazar la Ruta de la Seda y posibilitó, además, el ingreso del budismo tántrico a China (Ann Heirman, 2007).

El comercio se mantuvo casi sin interrupciones hasta el siglo IX en el marco del Imperio Uigur. Durante ese tiempo, China entregó enormes cantidades de seda a cambio de caballos.

Este comercio posibilitó un importante intercambio religioso y cultural ya que en la etnia Uigur habia budistas, nestorianos y maniqueos hasta que inician a partir del $742 \mathrm{~d}$. C. su conversión al Islam (Congreso Mundial Uigur, 2004).

Con la caída del imperio Uigur, el Islam comienza a expandirse con la instalación del Califato Islámico en Asia central y en el siglo X se producen conflictos que provocaron la interrupción de esta ruta y la casi desaparición del budismo (Foltz, 2010).

A principios del siglo XIII comienza a expandirse el imperio Mongol por todo el continente asiático, que logró consolidar estabilidad política, seguridad para los viajeros $\mathrm{y}$, al poner fin al monopolio del Califato Islámico sobre el comercio mundial, se restableció la Ruta de la Seda.

A la muerte de Gengis Kan (1227) la Ruta de la Seda quedó en manos de sus hijas y lo sucede su hijo Ogodei (Gengis Kan dejó así establecida la meritocracia al quitar privilegios de sangre o condición de nacimiento a sus hijos mayores, pues Ogodei era su hijo menor) (Veisaga, 2011).

Ogodei continuó con la expansión del imperio mediante la conquista de Polonia y Hungria hasta llegar a las puertas de Viena en 1242 y consolidó la jerarquía del Gran Kan sobre los diversos reinos mongoles (Götting, 2015).

En 1287, los mongoles envian a Rabban Bar Sauma como embajador frente a las cortes europeas. A partir de ahí y hasta fines del siglo XIV comienza un intenso tráfico comercial, cultural y religioso.

En ese período llegan por esta ruta numerosos misioneros cristianos a Oriente, como Guillermo de Rubruck, Benedicto de Polonia, Giovanni da Pian del Carpine y André de Longjumeau (quien en el primer viaje llevó cartas del Papa Inocencio IV y en el segundo, regalos y cartas de Luis IX de Francia para Güyük Kan).

A su retorno, estos misioneros traen la visión del mundo del budismo tántrico que inspiro a Gengis Kan y a sus continuadores y la difundieron en Europa. Esta visión inflamó la sed de conocimiento de Marco Polo en tiempos de Kublai Kan- (Polo, 2011) y de otros misioneros como Odorico de Pordenone, Giovanni de' Marignolli, Juan de Montecorvino y Niccolò de' Conti. 
A mediados del siglo XIV, la peste negra debilitó el poder político mongol establecido por Gengis Kan y su visión transteísta y protectora de todas las creencias y formas de vida (Hays J, 2005).

Esta visión transteísta (tántrica) se refugia en las tierras de Bothan, en el techo del mundo (Tíbet).

Los mongoles fueron convirtiéndose lentamente al Islam a partir de 1323 con el establecimiento de los sultanatos y se fue perdiendo la unidad política, cultural y la seguridad de la Ruta, que dejó de resultar una vía económica posible.

Finalmente, a mediados del siglo XV, la desaparición de la Ruta de la Seda propició la búsqueda, por parte de las potencias europeas, de nuevas rutas hacia el floreciente imperio chino - sobre todo las maritimas-.

Fue así que se dieron las exploraciones portuguesas del océano Índico, que incluyeron el mar de China, y que dieron lugar a la llegada del primer barco mercante europeo a las costas de China en 1513.

De este modo los portugueses consolidan fortificaciones en Macao y en Goa a donde habria de llegar como embajador Antonio de Montserrat en 1574.

\section{E1 tantra. La visión esotérica del Budismo}

Con la expansión del hinduismo, el budismo es desplazado de la India y se refugia entre las poblaciones de Asia central como la etnia Uigur en la actual Turquestán y se expande a lo largo de toda la Ruta de la Seda.

La combinación del budismo indio con influencias occidentales (grecobudismo) dio origen a diversas escuelas budistas en Asia Central y en China.

A fines del siglo VII ingresa la forma esotérica del budismo (tántrica) y se consolida en el siglo VIII en el Tíbet como budismo vajrayana.

Ésta es la forma de budismo que toman los gobernantes (de ahí la denominación de Tantra Real o transteísta), que promueve la igualdad completa de personas y género, la idea del sujeto como una construcción de la cultura (es decir, sin una esencia previa al momento de nacer y que condicionaría su estatus social o privilegios de casta) y la noción de deidades metafóricas -útiles para modelar el carácter de las personas pero de absoluta inexistencia - además del postulado budista de verdad 
relativa, es decir: la verdad es lo que ven los ojos de cada persona, la verdad absoluta no existe (Capra, 2000).

Entonces, con la consolidación sucesiva de los imperios Uigur, Chino y Mongol, con esta cosmovisión tántrica no teísta o más acertadamente transteísta y como reflejo de sus pilares de igualdad social, inexistencia del alma (esencia humana a priori) y verdad relativa se consolido la seguridad y el libre intercambio de bienes y conocimiento por toda la Ruta de la Seda entre China y Europa.

Es esta visión de sujetos no divididos en castas ni diferenciados por sangre lo que maravilla a de Montserrat al decir que los tibetanos "no tienen reyes entre sî" (Gómez, 2013) e inflama la avidez de quienes viajaron especialmente (a partir de los escritos de éste) a iniciarse en el budismo tántrico tibetano como los jesuitas Antonio de Andrade y Juan de Brito.

De este fructífero intercambio cultural y religioso (citado anteriormente) entre los misioneros católicos y la visión del budismo tántrico propiciado por la seguridad en el tránsito de la Ruta de la Seda se desarrolla en Castilla el movimiento de los Alumbrados (O'Malley, 1995) como una de las formas proteicas del tantrismo (de Mora Vaquerizo, 1988) para terminar extendiéndose también por toda Hispanoamérica (Jiménez Rueda, 1945).

Este movimiento queda incorporado en la Compañia de Jesús y de ahí el legado que recibimos en Occidente y que justifica que esta biografia, aunque enciclopédica, merezca ser insertada en este primer artículo como homenaje y punto de partida. 


\section{Antonio de Montserrat}

Nació en Vic, Barcelona en 1536 y murió en Goa, India en 1600. Fue un jesuita catalán formado en Portugal que en 1574 fue destinado a la misión de la colonia portuguesa de Goa, en la India, desde donde viajaría por buena parte del Asia Central y la península arábiga (Sociedad Geográfica Española, 1998).

Este viajero y erudito dejó constancia de sus viajes en cuatro manuscritos, de los que solo se conservan dos: el "Mongolicae Legationis Commentarius", en latín, y "Relaçam do Equebar, rei dos mogores", en portugués, referentes a su estancia en la corte del gran Kan Akbar (Sociedad Geográfica Española, 1998).

Hijo de una familia noble de Osona, estudió en Barcelona, donde entró en contacto con San Ignacio de Loyola.

Fascinado por la vida de los misioneros, entró en la Compañía de Jesús el 1558 y fue destinado a Portugal —donde fue ordenado sacerdote- en 1561.

Estudió en la Universidad de Coimbra, Lisboa, donde fue prefecto de San Roque, vicerrector del colegio de San Antonio y preceptor del rey Sebastián I de Portugal.

\section{Embajador en la corte del Gran Mogol}

En 1574, a la edad de 38 años, vio cumplido su sueño de convertirse en misionero cuando fue enviado, con 39 jesuitas más, a la misión de la colonia portuguesa de Goa, en la India.

Cinco años más tarde le fue encomendada la misión de integrarse en la embajada que debía presentarse a la corte del rey Mogol Akbar, con el encargo específico de registrar todo lo que sucediera durante el viaje.

La presencia de los sacerdotes había sido pedida expresamente por el mismo rey.

Los jesuitas portugueses interpretaron esto como la voluntad de Akbar de abrazar la fe cristiana, lo que en ningún caso fue así. 
De este modo, el 13 de diciembre de 1579, Antonio de Montserrat, junto con los jesuitas Rodolfo Acquaviva y Francisco Henríquez —un converso persa que hacía de intérprete - así como de un embajador de Akbar, partieron de la colonia portuguesa de Daman hacia la capital del Imperio Mogol.

La llegada del grupo a Fatehpur Sikri, la nueva capital fundada por Akbar, tuvo lugar el 4 de marzo de 1580 y allí permanecieron durante un año dedicados a diálogos y debates interreligiosos fomentados por el mismo rey Akbar con representantes de otras religiones como el Islam, el hinduismo, el jainismo, el cristianismo y el budismo tántrico tibetano (Casa Asia Barcelona, 2005).

Montserrat aprovechó su estancia para instruirse en la lengua persa y acabó ganándose la confianza de Akbar como lo demuestra el hecho de que lo nombrara tutor de su hijo Murad (Gómez, 2008).

Al cabo de un año, en el norte, estalló una revuelta encabezada por un hermanastro del rey que tenía el apoyo de algunos caudillos afganos.

\section{Expedición militar}

A petición de Akbar, Montserrat se incorporó a la expedición militar y lo acompañó durante toda la campaña, que duró hasta 1581.

Este viaje le permitió al jesuita tomar contacto con buena parte de los territorios del Imperio. Pudo visitar Delhi, el Himalaya, Himachal Pradesh, el Cachemira, el Punjab y las estribaciones del Tíbet hasta el Afganistán.

E1 fin del conflicto coincidió con el regreso a la embajada de Goa, una vez que los jesuitas constataron la nula voluntad del monarca de convertirse al cristianismo.

En septiembre de 1582 llegaron a la colonia portuguesa donde, durante seis años, Montserrat trabajó sobre sus notas con la intención de lograr una obra más compleja y detallada. 


\section{El cautiverio en Yemen}

En 1588 Antonio de Montserrat recibió la orden directa del rey español, Felipe II, de dirigirse a Etiopía para dar apoyo a los misioneros allí emplazados y sondear la posibilidad de lograr un acercamiento del cristianismo copto con la Iglesia de Roma.

E1 2 de febrero de 1589, Montserrat y su compañero Pedro Páez zarparon de Goa camuflados como mercaderes armenios.

El viaje se interrumpe cuando en Dhufar (Yemen) fueron traicionados por el comandante que los debía llevar a Etiopia, el cual los entregó al jefe de la ciudad, quien, a su vez, los envió a Haymes, ciudad del interior donde estaba la residencia del sultán de Hadhramaut.

Tras cuatro meses encerrados en la cárcel de esta ciudad, fueron enviados a Sanaa, sede del gobernador turco de Yemen, donde permanecieron hasta el 1595, año en que fueron trasladados al puerto de Moka, en el Mar Rojo, donde tuvieron que servir como remeros en galeras turcas durante unos meses hasta que Montserrat enfermó gravemente y fue recluido en la cárcel de la ciudad.

Finalmente, un año más tarde, estos dos jesuitas fueron devueltos a Goa, tras el pago de un rescate.

La salud de ambos era muy precaria tras casi siete años de cautiverio y, si bien Páez se pudo recuperar, Antonio de Montserrat murió en Salcete, cerca de Goa, donde habia un famoso convento jesuita, en marzo de 1600.

\section{Su obra y la influencia que sobre él ejerció el tantrismo}

En el mismo año de su muerte culminó la versión definitiva de su obra "Mongolicae Legationis Commentarius" y junto a ella, el diseño de su mapa del Himalaya, una auténtica joya cartográfica que abarca gran parte de la India y grandes extensiones de Afganistán y Pakistán.

En ella aparecen más de doscientos topónimos, accidentes geográficos resaltados en distintas tonalidades y coordenadas geográficas, reflejadas con sorprendente precisión, que tienen como referencia el ecuador y dibujan la linea del trópico de Cáncer con toda exactitud. Además de la cordillera del Himalaya, en la parte norte se 
distinguen otras cadenas montañosas cuya disposición parece coincidir con el Karakorum, el Hindú Kush y el Pamir.

La exactitud del mapa y lo acertado de sus descripciones es tal que mantendría su vigencia hasta hace relativamente poco tiempo. En el año 2002, Josep Lluís Alay, al investigar la figura de Antonio de Montserrat como misionero y explorador, descubre que la gente relacionada con el alpinismo ya lo conocía porque a él se le atribuía el primer mapa del Himalaya.

En la crónica escrita, sus textos reflejan de manera fidedigna todos aquellos detalles trascendentes a ojos de un occidental como la geografia, la historia, la cultura y la religión de las diferentes comunidades que llegó a conocer, pero también una de las grandes obsesiones que movieron a los religiosos cristianos a adentrarse en las vastas extensiones asiáticas.

En sus crónicas podemos leer:

"En el interior de estas sierras moran unos gentios que se llaman Botthant. Nunca se lavan las manos y dan como razón que no se debe ensuciar una cosa tan clara y hermosa como el agua. Son hombres blancos y gruesos, no muy altos de cuerpo, pelean a pie $y$ no tienen rey entre si. Viven de hacer fieltro $y$ vienen a venderlo a una ciudad de este lado que se llama Negarcot: $y$ bajan en junio, julio, agosto y septiembre; fuera de estos meses no pueden venir a causa de las nieves..."2

Podemos inferir de esta nota el asombro que habrá experimentado al conocer una cultura donde los hombres son iguales entre sí.

Es decir, carentes de alma preexistente que los diferencie entre reyes y súbditos o brahmanes y parias y que es el primer principio del budismo tántrico tibetano (Gómez, 2009).

La obsesión que impulsaba a los jesuitas era la búsqueda de antiguas cristiandades perdidas y seguir el rastro de la expansión del cristianismo hacia la Mesopotamia, Asia Menor y Extremo Oriente alentados por las crónicas de algunos viajeros medievales al descubrir diferentes comunidades, así como por la existencia de las iglesias copta, abisinia, armenia y maronita.

2 Esta es la primera descripción que Occidente tiene del pais de Botthant, el mitico Tíbet. (Gómez, 2008) 
Roma buscaba desesperadamente pruebas de la existencia de un imperio a caballo entre la historia y la leyenda, un imperio dirigido por un rey-sacerdote poderosísimo, defensor de la fe cristiana ante el avance musulmán, el reino del Preste Juan en Etiopia.

Este rey, a mi entender, no sería otro que el propio Akbar.

Un año después de la muerte de Antonio Montserrat, en enero de 1601, el jesuita Antonio de Andrade llegó a Goa con el objetivo de emplazar una misión y buscar conocimiento en aquel misterioso reino aislado llamado Bottan o Tebat (Tíbet), lo que hace pensar que la crónica de Montserrat fue tenida en cuenta por los responsables de la Compañia de Jesús para incorporar el tantrismo -en su forma proteica de Alumbrados primero y más tarde Teología de la liberación- dentro de sus enseñanzas y prácticas.

\section{Conclusiones}

Produce vértigo pensar que el Gran Kan Mogol Yalaluddin Muhammad, Akbar, no llegara siquiera a sospechar que el viaje del jesuita y sus escritos tuvieran un significado tan trascendental para Occidente.

De alguna manera la invitación del emperador a los religiosos abriría la puerta al descubrimiento de uno de los últimos espacios a conquistar por los adalides de la fe cristiana, pero también cambiaría la cosmovisión de los viajeros de aquella incipiente Europa renacentista que indudablemente influenció en su modo de comprender el mundo y al hombre (Gómez, 2013).

Esta filosofia tántrica, que fue transmutando su nombre con los años $^{3}$, es la que transitamos en este momento como paradigma en occidente.

Y este modelo paradigmático tántrico o existencialista, es el que da lugar a la construcción de un sujeto, sujeto a una forma particular de

\footnotetext{
${ }^{3}$ De la misma manera que en oriente, por diversas circunstancias, tomó diversos nombres como taoísmo, sufismo, cábala (Buber, 2007) y finalmente revolución cultural en China (Chhaya, 2009) el tantrismo tomó en occidente la denominación de alumbrados, iluminismo, existencialismo, fenomenologia, teología de la liberación, surrealismo (Foljambe, 2008) y postmodernidad.
} 
experimentar el mundo, relacionarse con él y actuar. Es decir: la filosofia tántrica, sin que tengamos consciencia, es la que estaria determinando los valores morales e intereses de las generaciones recientes.

Sin embargo, tras aquel primer impacto, la obra de Montserrat permaneció en el anonimato durante varios siglos.

En 1906 Henry Hosten, un jesuita del Raj Británico especializado en la historia del cristianismo en la India, redescubrió el "Mongolicæ legationis Commentarius" y lo publicó en 1914.

La obra despertó un gran interés en los indólogos de la época.

En la actualidad, en pleno siglo XXI, la obra del jesuita está alcanzando el lugar que le corresponde por derecho propio gracias a la edición popular de sus obras traducidas del latín al castellano y al catalán por el orientalista Josep Luis Alay.

No obstante, aún quedan otros manuscritos redactados por Montserrat que hacen referencia a las costumbres y la geografia de la India y Asia Central sobre los que se desconoce su paradero.

E1 misterio de su ingente obra perdura hasta nuestros dias y espero que este artículo sirva para rescatarlos y traerlos a la historia conocida. 


\section{Referencias}

Ann Heirman, S. P. (2007). The Spread of Buddhism. Boston: Zürcher Oberland. Retrieved from https://books.google.es /

Bellec, F. (2003). Marchands au long cours. París: du Chêne-Hachette.

Buber, M. (2007). Eclipse de Deus: Consideracoes sobre la relacao entre religiao e filosofía. Verus.

Capra, F. (2000). El Tao de la Física. Sirio.

Casa Asia Barcelona. (2005). Programa Antoni de Montserrat 2005. Convocatoria pública de CA. Retrieved 2015, from http: / /www.casaasia.es/pdf/ 1606041139191087378759579.pdf

Chhaya, M. (2009). Dalai Lama. Hombre, monje, mistico. Grijalbo.

Congreso Mundial Uigur. (2004). Breve historia de Turkestán Oriental. Alemania: Primera Asamblea General del WUC. Retrieved from http: / / www.uyghurcongress.org/es /?cat=132

de Montserrat, A. (2005). Embajador en la corte del Gran Mogol. Viajes de un jesuita catalán del siglo XVI por la India, Pakistán, Afganistán y el Himalaya (segunda ed.). (J. L. ALAY, Trans.) Lérida.

de Montserrat, A. (n.d.). The Commentary of Father Monserrate, donde his journey to the court of Akbar. (1. SN Banerjee. Oxford University Press, Ed., \& T. d. Hoyland, Trans.)

de Mora Vaquerizo, J. M. (1988). Tantrismo Hindú y Proteico. Universidad Nacional Autónoma de México.

Foljambe, A. (2008). Tantric Buddism, desire, and the bodyin surrealism and Georges Bataille. University of Manchester.

Foltz, R. (2010). Religions of the Silk Road: Premodern Patterns of Globalization (2 ed.). Palgrave Macmillan.

Gómez, O. R. (2008). Manual de Tantra ...desde el tantra a la Tecnología del deseo (Primera ed.). (F. MenteClara, Ed.) Buenos Aires, Argentina: MenteClara.

Gómez, O. R. (2009). Manual de Tantra - Reprogramación emocional (Tercera ed.). (F. MenteClara, Ed.) Buenos Aires, Argentina: Ediciones MenteClara.

Gómez, O. R. (2013). El tantrismo dentro de la Compañía de Jesús - del Tíbet al Vaticano hoy (Primera ed.). (F. MenteClara, Ed.) Buenos Aires, Argentina: Ediciones Menteclara.

Götting, D. (2015). 1241: Os mongóis chegam à Europa. Deutsche Welle.

Hays J, N. (2005). Epidemics and Pandemics: Their Impacts on Human History. ABC Clio. 
Jiménez Rueda, J. (1945). La secta de los alumbrados en la Nueva España (Vols. Tomo XVI, Numeral 1). Mexico: Boletín del Archivo General de la Nación.

O'Malley, J. W. (1995). Los primeros jesuitas. SAL TERRAE.

Polo, M. (2011). La Ruta de la Seda. El arte del Viaje. Un italiano en la corte de Kublai Kan. Blume.

Sociedad Geográfica Española. (1998). Antonio de Montserrat: En la última frontera. Boletín $N^{o} 43$ de la SGE. Retrieved 2015, from http:/ /www.sge.org/sociedadgeografica-espanola/publicaciones/boletines / numeros-publicados/boletinno-43/antoni-de-montserrat-en-la-ultima-frontera.html

Urban, H. B. (1999). The extreme orient: the construction of 'tantrism'as a category in the orientalist imagination. (Vols. Religion, 29(2), 123-146.). Retrieved from https: / /www.academia.edu/4527418/The_Extreme_Orient_The_Construction _of_Tantrism_as_a_Category_in_the_Orientalist_Imagination

Veisaga, R. (2011). Imperios e Historia. Trafford Publishing. 


\section{Bibliografia}

Ann Heirman, S. P. (2007). The Spread of Buddhism. Boston: Zürcher Oberland. Obtenido de https:// books.google.es /

Bellec, F. (2003). Marchands au long cours. París: du Chêne-Hachette.

Buber, M. (2007). Eclipse de Deus: Consideracoes sobre la relacao entre religiao e filosofía. Verus.

Capra, F. (2000). El Tao de la Física. Sirio.

Casa Asia Barcelona. (2005). Programa Antoni de Montserrat 2005. Convocatoria pública de CA. Recuperado el 2015, de http: / /www.casaasia.es/pdf/ 1606041139191087378759579.pdf

Chhaya, M. (2009). Dalai Lama. Hombre, monje, mistico. Grijalbo.

Congreso Mundial Uigur. (2004). Breve historia de Turkestán Oriental. Alemania: Primera Asamblea General del WUC. Obtenido de http:/ / www.uyghurcongress.org/es /?cat=132

de Montserrat, A. (2005). Embajador en la corte del Gran Mogol. Viajes de un jesuita catalán del siglo XVI por la India, Pakistán, Afganistán y el Himalaya (segunda ed.). (J. L. ALAY, Trad.) Lérida.

de Montserrat, A. (s.f.). The Commentary of Father Monserrate, donde his journey to the court of Akbar. (1. SN Banerjee. Oxford University Press, Ed., \& T. d. Hoyland, Trad.)

de Mora Vaquerizo, J. M. (1988). Tantrismo Hindú y Proteico. Universidad Nacional Autónoma de México.

Foljambe, A. (2008). Tantric Buddism, desire, and the bodyin surrealism and Georges Bataille. University of Manchester.

Foltz, R. (2010). Religions of the Silk Road: Premodern Patterns of Globalization (2 ed.). Palgrave Macmillan.

Gómez, O. R. (2008). Manual de Tantra ...desde el tantra a la Tecnología del deseo (Primera ed.). (F. MenteClara, Ed.) Buenos Aires, Argentina: MenteClara.

Gómez, O. R. (2009). Manual de Tantra - Reprogramación emocional (Tercera ed.). (F. MenteClara, Ed.) Buenos Aires, Argentina: Ediciones MenteClara.

Gómez, O. R. (2013). El tantrismo dentro de la Compañía de Jesús - del Tíbet al Vaticano hoy (Primera ed.). (F. MenteClara, Ed.) Buenos Aires, Argentina: Ediciones Menteclara.

Götting, D. (2015). 1241: Os mongóis chegam à Europa. Deutsche Welle.

Hays J, N. (2005). Epidemics and Pandemics: Their Impacts on Human History. ABC Clio.

Jiménez Rueda, J. (1945). La secta de los alumbrados en la Nueva España (Vols. Tomo XVI, Numeral 1). Mexico: Boletín del Archivo General de la Nación. 
O'Malley, J. W. (1995). Los primeros jesuitas. SAL TERRAE.

Polo, M. (2011). La Ruta de la Seda. El arte del Viaje. Un italiano en la corte de Kublai Kan. Blume.

Sociedad Geográfica Española. (1998). Antonio de Montserrat: En la última frontera. Boletin $N^{\circ} 43$ de la SGE. Recuperado el 2015, de http:/ / www.sge.org/sociedad-geograficaespanola/publicaciones / boletines / numeros-publicados/boletin-no43/antoni-de-montserrat-en-la-ultima-frontera.html

Urban, H. B. (1999). The extreme orient: the construction of 'tantrism'as a category in the orientalist imagination. (Vols. Religion, 29(2), 123-146.). Obtenido de https: / /www.academia.edu/4527418/The_Extreme_Orient_The_Construction _of_Tantrism_as_a_Category_in_the_Orientalist_Imagination

Veisaga, R. (2011). Imperios e Historia. Trafford Publishing. 